\title{
A Statistical Approach to Confined Seepage Problem with Generation of Random Permeability Field
}

\author{
Noriyuki Kobayashi ${ }^{1}$, Yoshitaka Yoshitake ${ }^{2}$ and Kenji Iima ${ }^{3}$
}

\begin{abstract}
In many design practices for rehabilitation or construction of earthen embankments, a deterministic approach is employed for seepage flow analysis, assuming hydrogeological homogeneity of embankment soils. Since in fact soil permeability is heterogeneous, such a conventional approach may often mislead engineers into making an inadequate design with less structural safety and less economical efficiency. This paper intensively investigates reliability of seepage analysis based on a statistical approach that can consider spatial variations of soil permeability. First, random fields with mean, standard deviation and spatial correlation structure are generated, and then finite element seepage analysis in these random fields is performed. The statistical analyses are then implemented through the procedure of Monte Carlo simulation. The statistical solutions to a confined seepage flow problem are compared with the theoretical solution based on deterministic approach. As a result, the mean flow rate $Q_{m}$ becomes far from the theoretical value $Q_{t}$ as the global standard deviation $\sigma_{k}$ and/or the correlation distance $a$ increase. The spatial correlation structure, not to mention the mean and the standard deviation of permeability, is shown to have a significant effect on seepage flow rate.
\end{abstract}

Keywords: Statistics; Spatial correlation; Monte Carlo simulation; Seepage analysis

\section{Introduction}

When an overage irrigation tank is rehabilitated, design and construction of its embankment are carried out based on uncertain information, because it is difficult to get the design documents having been made in those days and/or only a narrow spectrum of investigation is made on account of restricted time, economy and physical condition. So, when the behavior of embankment of the tank is analyzed, spatial distribution of the materials must be estimated by small number of data obtained from such limited investigation. Thus in analyzing seepage flow in the embankment, for example, there is often no choice but to make an assumption of hydrogeologically homogeneous embankment, i.e., to use a particular value of permeability that can be evaluated by simply averaging its in situ values. Employing the results, obtained under this assumption, in design practice may for the worst result in losing the safety and economical efficiency in rehabilitation or construction of the embankment. In this context, it is an essential need to develop a methodology to deduce a plausible distribution of the permeability inside the embankment from limited information or data available. Undoubtedly a useful methodology is to deal with the permeability in a statistical sense.

Over the past few decades, availability of applying first and second moment statistics (mean, standard deviation and spatial correlation) to design has been recognized and a considerable number of studies have been made on statistically generating spatially heterogeneous permeability field (e.g., Freeze (1975)

\footnotetext{
${ }^{1}$ Research Associate, Faculty of Agriculture, Ehime University, 3-5-7 Tarumi, Matsuyama, Ehime, 790-8566 Japan

${ }^{2}$ Professor, Faculty of Agriculture, Ehime University, 3-5-7 Tarumi, Matsuyama, Ehime, 790-8566 Japan

${ }^{3}$ M. Sc. Student, Faculty of Agriculture, Ehime University, 3-5-7 Tarumi, Matsuyama, Ehime, 790-8566 Japan
}

and Smith and Freeze (1979) for 1-D groundwater flow, Griffiths and Fenton (1993) and Fenton and Griffiths (1996) for 2-D flow beneath water retaining structures and through earth dam, and Griffiths and Fenton (1998) for 3-D beneath water retaining structures.)

This paper aims at detailed investigation of flow realizations that result from a statistical approach to confined seepage problem under uncertainty of soil permeability distribution. First, random fields with mean, standard deviation and spatial correlation structure are generated, and finite element seepage analysis in these random fields is performed. The statistical results are then evaluated by using the procedure of Monte Carlo simulation. The statistical results obtained are compared with each other and with exact solution obtained by deterministic approach. As a result, the spatial correlation structure of permeability is shown to have a significant effect on flow rates.

\section{Generation of random field of soil perme- ability}

The random soil permeability fields are generated taking the following process.

1. A Gaussian random field, $G(x, y)$, is generated with mean, variance and spatial correlation distance.

2. The permability coefficient is assumed to follow a lognormal distribution, and the Gaussian random numbers gained at the first stage are transformed lognormally.

\subsection{Process for generation of Gaussian ran- dom field}

When the permeability coefficient is spatially distributed, there is some correlation between its values 


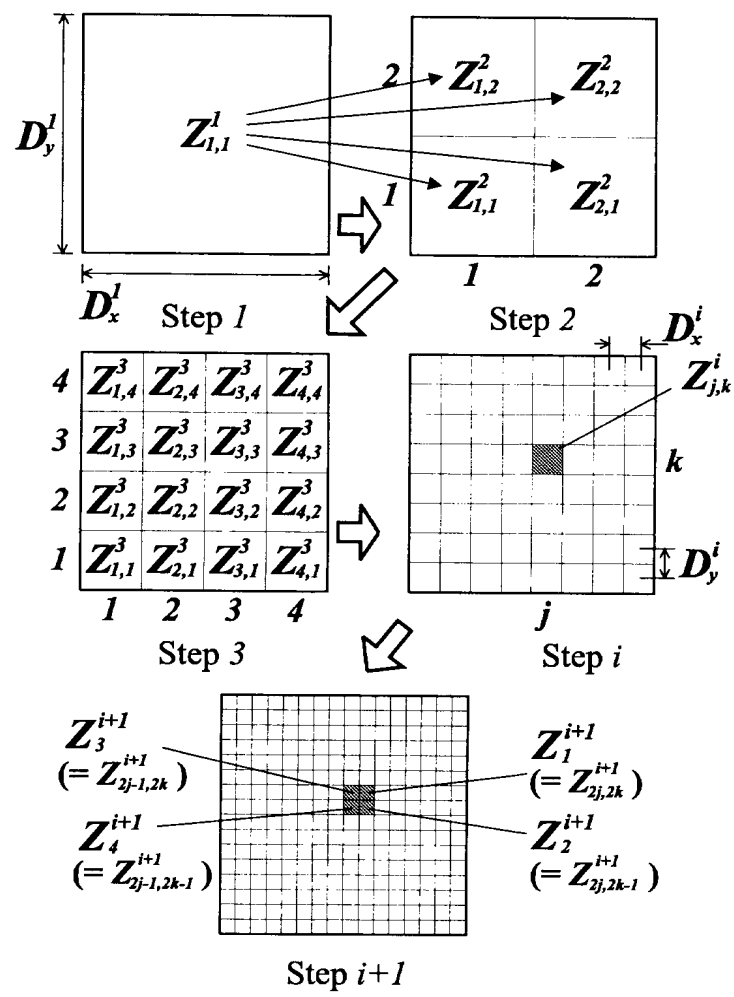

Figure 1: Process for generation of random number

at any two points in the field. The permeabilities at any two points are almost the same if the points of interest are closely located. Otherwise, correlation is reduced with the increasing distance between the points.

The structure of spatial distribution for soil permeability can be represented by a covariance function that describes the variability of permeability between two points. The covariance function employed herein is an exponential type in two dimensions expressed in the following form (Journel (1978), Hamaguchi (1998)):

$$
C\left(h_{x}, h_{y}\right)=\sigma_{g}^{2} \exp \left\{-\sqrt{\left(\frac{h_{x}}{a_{x}}\right)^{2}+\left(\frac{h_{y}}{a_{y}}\right)^{2}}\right\}
$$

where $C(\cdot)$ : covariance function, $\sigma_{g}$ : global standard deviation, $h_{x}, h_{y}$ : separated distance between a pair of any two points in the $x$ and $y$ directions, respectively, $a_{x}, a_{y}$ : correlation distance in the $x$ and $y$ directions, respectively, and the subscripts $x, y$ : Cartesian coordinates.

A Gaussian random number $Z$ is generated with the covariance function (1) and is defined as the average of $G(x, y)$ over the intervals $s \leq x \leq t$ and $u \leq y \leq v$ :

$$
Z=\frac{1}{(t-s)(v-u)} \int_{s}^{t} \int_{u}^{v} G(x, y) d x d y
$$

The process for generation of $Z$ is illustrated in Figure 1. A whole domain where $Z$ is generated is a

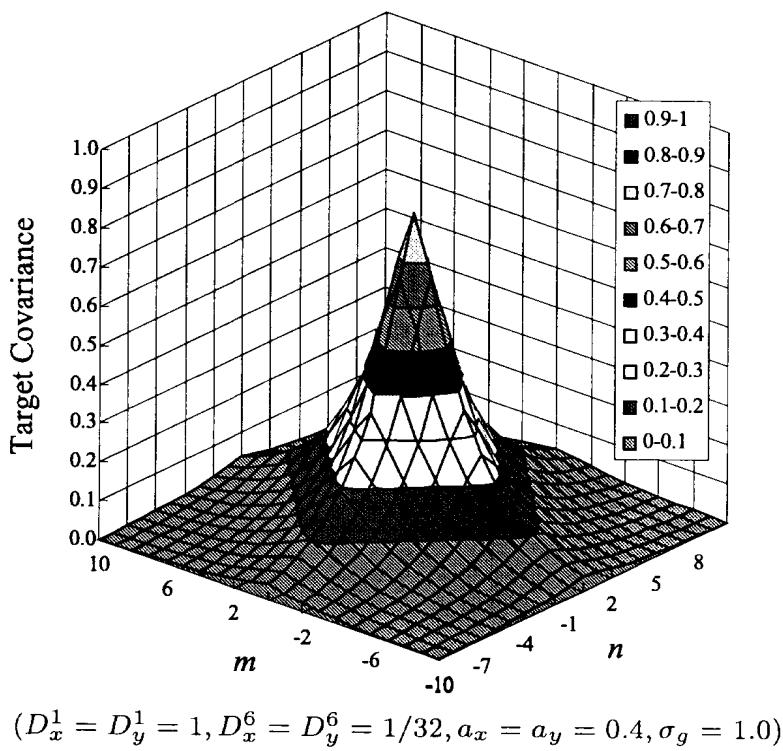

Figure 2: Distribution of target covariance

rectangle with $D_{x}^{1}$ in width and $D_{y}^{1}$ in length. First, a global mean $\mu_{g}$ for the domain is defined at Step 1. At Step 2, the domain is subdivided into 4 equal cells subject to the rule that their values must average to $\mu_{g}$ and be correlated with each other according to (1). At the subsequent step, each parent cell generated at the previous step is subdivided into 4 equal cells due to the same rule that their values average to the parent mean (Fenton and Vanmarcke (1990)).

The covariance between a pair of cells separated by $m$ cells in $x$ direction and $n$ cells in $y$ direction is called target covariance and is represented as follows:

$$
\begin{aligned}
E\left[Z_{j, k}^{i} Z_{j+m, k+n}^{i}\right] & E\left[\left(\frac{1}{D_{x}^{i} D_{y}^{i}}\right) \int_{(j-1) D_{x}^{i}}^{j D_{x}^{i}} \int_{(j+m-1) D_{x}^{i}}^{(j+m) D_{x}^{i}}\right. \\
= & \left.\int_{(k-1) D_{y}^{i}}^{k D_{y}^{i}} \int_{(k+n-1) D_{y}^{i}}^{(k+n) D_{y}^{i}} G(x, y) G\left(x^{\prime}, y^{\prime}\right) d x d x^{\prime} d y d y^{\prime}\right] \\
= & \left(\frac{1}{D_{x}^{i} D_{y}^{i}}\right) \int_{0}^{D_{x}^{i}} \int_{m D_{x}^{i}}^{(m+1) D_{x}^{i}} \int_{0}^{D_{y}^{i}} \int_{n D_{y}^{i}}^{(n+1) D_{y}^{i}} \\
& C\left(x-x^{\prime}, y-y^{\prime}\right) d x d x^{\prime} d y d y^{\prime}
\end{aligned}
$$

where $Z_{j, k}^{i}$ : Gaussian random number of the $j, k$ th cell at Step $i, E[\cdot]$ : expectation operator, $D_{x}^{i}, D_{y}^{i}$ : length of a cell in $x, y$ coordinate directions at Step $i$, respectively, $h_{x}=m D_{x}^{i}, h_{y}=m D_{y}^{i}, C(\cdot)$ : covariance function (1), and the superscript $i$ : step number. Figure 2 illustrates a sample distribution of target covariance (3) with $i=6, D_{x}^{1}=D_{y}^{1}=1, D_{x}^{6}$ $=D_{y}^{6}=1 / 32, a_{x}=a_{y}=0.4$, and $\sigma_{g}=1.0$.

Gaussian random number in each cell at Step $i+1$ 


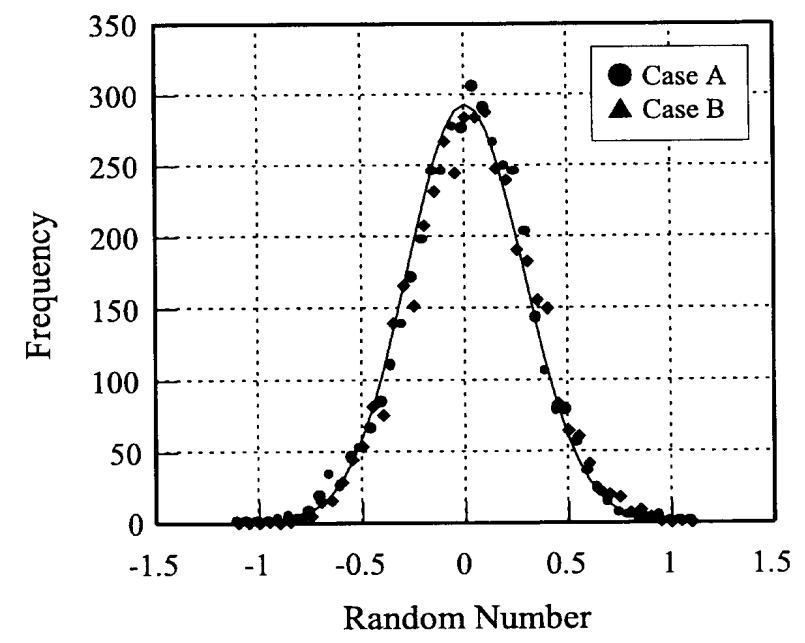

Figure 3: Frequency distribution of $Z$ after 7 steps

is generated by using (4) over a whole domain. On the right hand side of the first formula in (4), the second term can be obtained by a combination of $Z$ values at Step $i$ in some neighborhood cells.

$$
\left.\begin{array}{l}
Z_{s}^{i+1}=\sum_{r=1}^{s} c_{s, r}^{i+1} U_{r, j, k}^{i+1}+\sum_{l=1}^{n_{x y}} \alpha_{l, s}^{i} Z_{m(l), n(l)}^{i} \\
s=1,2,3 \\
Z_{4}^{i+1}=4 Z_{j, k}^{i}-\sum_{s=1}^{3} Z_{s}^{i+1}
\end{array}\right\}
$$

where $Z_{s}^{i+1}(s=1,2,3,4)$ : random numbers at Step $i+1$ for 4 cells produced by subdividing $Z_{j, k}^{i}, n_{x y}$ : number of neighborhood cells of $Z_{j, k}^{i}\left(n_{x y}=\left(2 n_{x}+\right.\right.$ $\left.1) \times\left(2 n_{y}+1\right)\right), m(l)$ and $n(l)$ : indexing functions prescribing the positions of the neighborhood cells, and $U$ : a Gaussian variate with zero mean and unit variance. The coefficients $\alpha_{l, s}^{i}$ and the component $c_{s, r}^{i+1}$ of the coefficient matrix in (4) can be calculated from the following formulae.

$$
\begin{array}{r}
E\left[Z_{s}^{i+1} Z_{m(p), n(p)}^{i}\right]=\sum_{l=1}^{n_{x y}} \alpha_{l s}^{i} E\left[Z_{m(l), n(l)}^{i} Z_{m(p), n(p)}^{i}\right] \\
s=1,2,3 \quad p=1,2, \cdots, n_{x y}
\end{array}
$$

$$
\begin{aligned}
\sum_{t=1}^{3} c_{r, t}^{i+1} \cdot c_{s, t}^{i+1}= & E\left[Z_{r}^{i+1} Z_{s}^{i+1}\right] \\
& -\sum_{l=1}^{n_{x y}} \alpha_{l, r}^{i} E\left[Z_{m(l), n(l)}^{i} Z_{s}^{i+1}\right]
\end{aligned}
$$

\subsection{Comparison of random fields}

Different two random fields are comparatively investigated considering two values of isotropic correlation distance; $a\left(a_{x}=a_{y}\right)=0.4$ and 0.0 that are herein

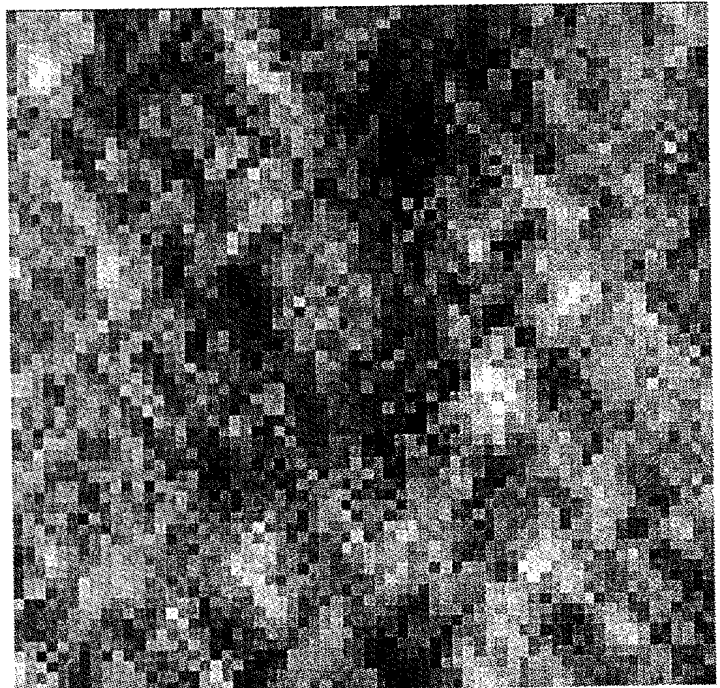

(a) Case A

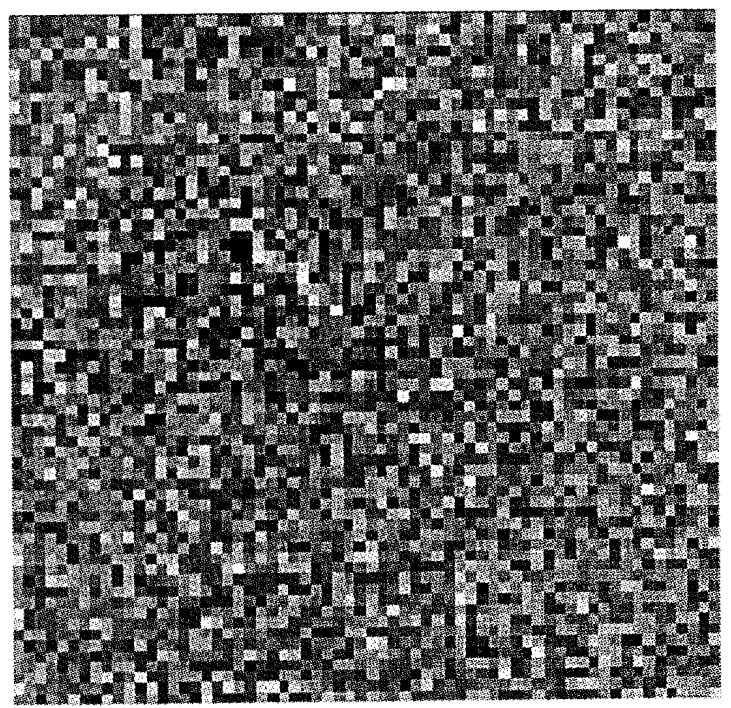

(b) Case B

Figure 4: Spatial distribution of $Z$ after 7 steps

referred to as Case A and Case B, respectively. For this, a square domain with $D_{x}^{1}=D_{y}^{1}=1$ is considered, and the global mean $\left(\mu_{g}=0.0\right)$ and the global standard deviation $\left(\sigma_{g}=0.25\right)$ are taken as the same in both cases. Random number genaration is made up 7 steps to have a total of $4,096(64 \times 64)$ random numbers.

The frequency and the spatial distributions of Gaussian random number $Z$ are shown in Figure 3 and 4 , respectively. In Figure 3 , the solid line is Gaussian probability density function which has the same mean and standard deviation. The frequency distributions are indistinguishably different between both cases. As is clear from Figure 4, however, the random numbers generated in Case A are strongly correlated in space compared with those in Case B. 


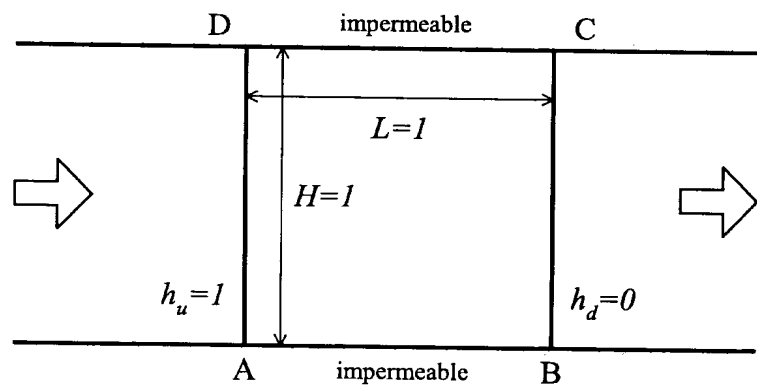

Figure 5: Difinition sketch of confined flow model

\subsection{Transformation of Gaussian random field into permeability field}

There is a large number of studies for direct and/or indirect evidence to support the statement that the probability density function for soil permeability coefficient is $\log$ normal (e.g., Freeze (1975), Hoeksema and Kitanidis (1985) and Sudicky (1986)).

The random permeability field is obtained through the following transformation.

$$
k_{j, k}=\exp \left(\mu_{\ln k}+\sigma_{\ln k} Z_{j, k}^{i}\right)
$$

where $k_{j, k}$ : permeability coefficient of the $j, k$ th cell, and $\mu_{\ln k}, \sigma_{\ln k}$ : global mean and standard deviation of the logarithm of permeability coefficient $k$, respectively, and $Z_{j, k}^{i}$ : Gaussian random number of the $j, k$ th cell at Step $i$. The values of $\mu_{\ln k}$ and $\sigma_{\ln k}$ can be obtained from (8) and (9).

$$
\begin{gathered}
\mu_{\ln k}=\ln \left(\mu_{k}\right)-\frac{1}{2} \sigma_{\ln k}^{2} \\
\sigma_{\ln k}^{2}=\ln \left(1+\frac{\sigma_{k}^{2}}{\mu_{k}^{2}}\right)
\end{gathered}
$$

where $\mu_{k}, \sigma_{k}$ : global mean and global standard deviation of permeability coefficient, respectively.

\section{Statistical analysis for seepage problem}

\subsection{Procedure of Monte Carlo simulation}

In this study, Monte Carlo simulation is performed to estimate reliability of steady flow analysis in the random permeability field, taking the following procedure.

1. A global mean $\mu_{k}$, a global standard deviation $\sigma_{k}$ and a correlation distance $a\left(a_{x}=a_{y}\right)$ are given and a set of random numbers $Z$ in a whole domain is generated by (4).

2. Random permeability field is obtained through the transformation by (7) to (9).

3. Flow rate $Q$ is calculated by $2-\mathrm{D}$ finite element analysis for the random permeability field. The elements used here are 4-node quadrilaterals and are coincided with the cells generated for random permeability field.

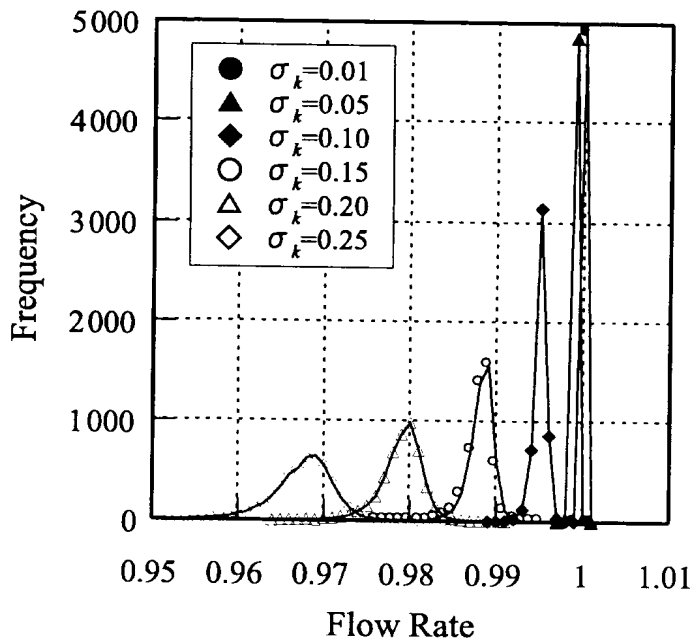

Figure 6: Frequency distribution of flow rate $(a=0.4)$

4. 5,000 sets of random numbers with the same value of $\mu_{k}, \sigma_{k}$ and $a$ at step 1 are generated and the procedures from step 2 to 3 are performed for each set.

5. Frequency distributions of $Q$, mean flow rate $Q_{m}$ and variance of flow rate $\sigma_{f}^{2}$ are obtained.

6. $\sigma_{k}$ and $a$ in step 1 are changed and the procedures from step 2 to 5 are repeated.

\subsection{Governing equation and boundary condi- tions}

As touched on in the previous subsection, 2-D finite element analysis is performed to analyze heterogeneous seepage problems. The governing equation for 2 -D steady flow in a saturated porous medium can be written in the form:

$$
\frac{\partial}{\partial x}\left(k_{x} \frac{\partial h}{\partial x}\right)+\frac{\partial}{\partial y}\left(k_{y} \frac{\partial h}{\partial y}\right)=0
$$

where $k_{x}$ and $k_{y}$ : coefficients of permeability in the $x$ and $y$ directions, respectively, and $h$ : total head. In this paper, the permeability field is assumed to be isotropic $\left(k=k_{x}=k_{y}\right)$.

The general expressions for boundary conditions are give by:

$$
\begin{array}{ll}
h=\bar{h} & \text { on } B_{1} \\
-k \frac{\partial h}{\partial n}=\overline{V_{n}} & \text { on } B_{2}
\end{array}
$$

where $B_{1}$ : the boundary where $h$ is prescribed as $\bar{h}$ (Dirichlet type), $B_{2}$ : the boundary where the outward fluid flux is prescribed as $\overline{V_{n}}$ (Neumann type).

\subsection{Simulation model}

To compare with the theoretical solution by deterministic approach, a confined flow model is considered. 
Table 1: Statistics of flow rate $Q$ for confined flow model $(a=0.4)$

\begin{tabular}{c|cccccc}
\hline$\sigma_{k}$ & $\begin{array}{c}\text { mean } \\
\text { value } Q_{m}\end{array}$ & $\begin{array}{c}\text { standard } \\
\text { deviation } \sigma_{f}\end{array}$ & $\begin{array}{c}\text { variance } \\
\sigma_{f}^{2}\end{array}$ & $\begin{array}{c}\text { coefficient of } \\
\text { variation } \sigma_{f} / Q_{m}\end{array}$ & $\begin{array}{c}\text { minimum } \\
\text { value } Q_{\min }\end{array}$ & $\begin{array}{c}\text { maximum } \\
\text { value } Q_{\max }\end{array}$ \\
\hline 0.01 & 1.0000 & $6.69 \times 10^{-6}$ & $4.47 \times 10^{-11}$ & $6.69 \times 10^{-6}$ & 0.9999 & 1.0000 \\
0.05 & 0.9986 & $1.69 \times 10^{-4}$ & $2.85 \times 10^{-8}$ & $1.69 \times 10^{-4}$ & 0.9973 & 0.9993 \\
0.10 & 0.9945 & $6.70 \times 10^{-4}$ & $4.49 \times 10^{-7}$ & $6.74 \times 10^{-4}$ & 0.9892 & 0.9973 \\
0.15 & 0.9877 & $1.49 \times 10^{-3}$ & $2.21 \times 10^{-6}$ & $1.51 \times 10^{-3}$ & 0.9760 & 0.9939 \\
0.20 & 0.9785 & $2.59 \times 10^{-3}$ & $6.71 \times 10^{-6}$ & $2.65 \times 10^{-3}$ & 0.9581 & 0.9892 \\
0.25 & 0.9670 & $3.95 \times 10^{-3}$ & $1.56 \times 10^{-5}$ & $4.08 \times 10^{-3}$ & 0.9358 & 0.9833 \\
\hline
\end{tabular}

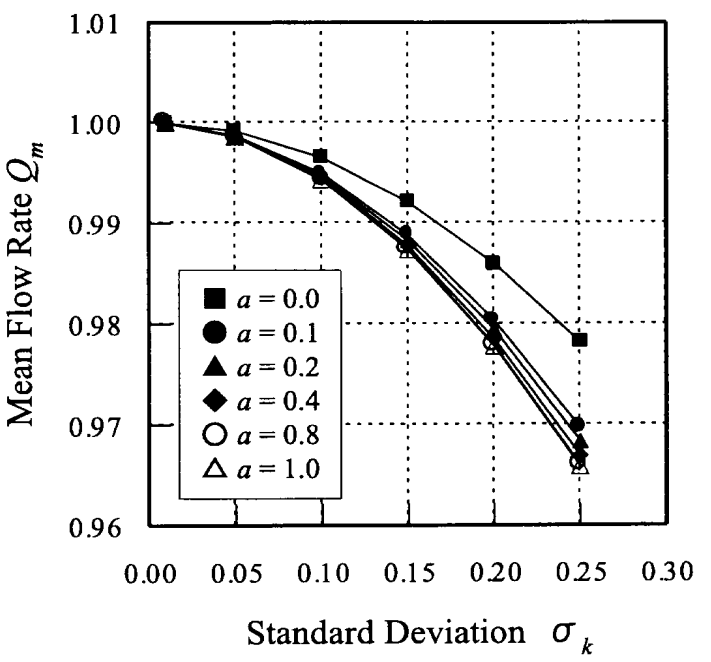

(a) mean

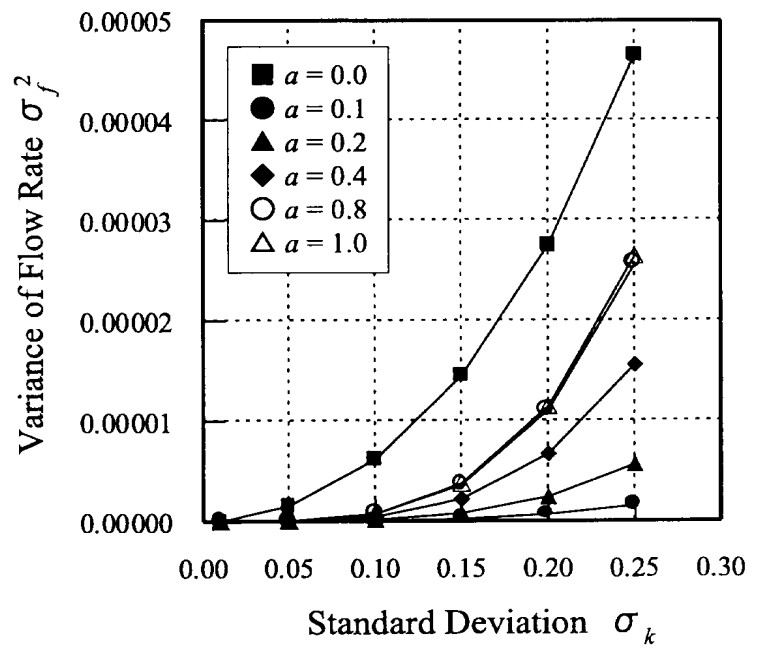

(b) variance

Figure 7: Relationship between $\sigma_{k}$ and statistics of flow rate

Figure 5 shows the confined flow model. The domain for the analysis is made dimensionless to be 1.0 in height $(H)$ and 1.0 in width $(L)$, and the total heads at its upstream and downstream boundaries are taken as $h_{u}=1.0$ and $h_{d}=0.0$, respectively. The permeability coefficient is given by (7), and $Z$ is generated with a fixed value of $\mu_{k}=1.0$ and various values of $\sigma_{k}$ and $a\left(a_{x}=a_{y}\right)$.

The parametric studies are designed to show the effect of $\sigma_{k}$ and $a$ on $Q$. The values $\sigma_{k}=0.01,0.05$, $0.10,0.15,0.20$ and 0.25 , and $a=a_{x}=a_{y}=0.0,0.1$, $0.2,0.4,0.8$ and 1.0 are considered. Generation of random numbers are made up to 6 steps $(i=6)$ to have 1,024 cells. The domain is thus devided into 1,024 finite elements with 1,089 nodes.

The theoretical solution is represented by the following equation.

$$
Q_{t}=k \cdot H \cdot\left(h_{u}-h_{d}\right) / L
$$

The dimensionless coefficient of permeability is given by $k=1.0$ correlated with $\mu_{k}=1.0$, and therefore the theoretical total flow rate $Q_{t}$ is 1.0 .

\section{Results and discussion}

To investigate fundamental tendency of this method, a frequency distribution of $Q$ with $a=0.4$ is shown in Figure 6 and these results are summarized in Table 1. For small values of $\sigma_{k}$, that is, for $\sigma_{k}=0.01,0.05$, $Q_{m}$ is almost equal to $Q_{t}$ and $\sigma_{f}^{2}$ is very small. These two curves have a sharper summit and a narrower foot than that of other curves (Figure 6). On the contrary, as $\sigma_{k}$ increases, $Q_{m}$ becomes much smaller than $Q_{t}$ and $\sigma_{f}^{2}$ becomes much larger than that of smaller values of $\sigma_{k}$.

Figure 7 shows influence of $a\left(a_{x}=a_{y}\right)$ on statistics of $Q$. As $\sigma_{k}$ increases, $Q_{m}$ decreases. And, as $a$ increases, $Q_{m}$ tends to become far from $Q_{t}$, or its reduction rate becomes larger (Figure $7(\mathrm{a})$ ). In the case of no spatial correlation $(a=0.0)$, the reduction rate of $Q_{m}$ is the smallest among all cases considered. In order to demonstrate the reduction of flow rate with the increasing $\sigma_{k}$, typical two permeability distributions, selected from 5,000 distributions generated with $\sigma_{k}=0.25$, are shown in the insert of Figure 8 in which the cells are gradated from black to white as the value of permeability coefficient is increased. As $\sigma_{k}$ becomes larger, the portion of lower permeability becomes dominant. It is further expected that lower permeability cells would concentrate in a particular part and be arranged like an impervious wall. And the flow pattern is somewhat like that of hor- 

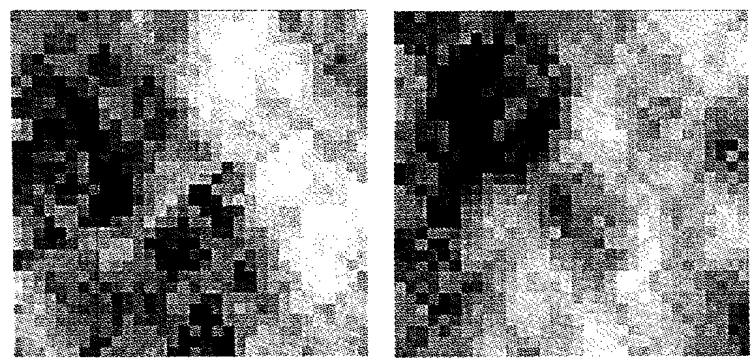

$\left(D_{x}^{1}=D_{y}^{1}=1, D_{x}^{6}=D_{y}^{6}=1 / 32, a_{x}=a_{y}=0.4, \sigma_{k}=0.25\right)$

Figure 8: Typical spatial distributions of permeability coefficient

izontal flow through an isotropic vertically layered system which has some layers of lower permeability, so that the existence of lower permeability layers in such a system may have a significant effect on flow rate reduction.

A few particular features can be found in Figure $7(\mathrm{~b})$. The curves of $\sigma_{f}^{2}$ seem to be a quadratic function of $\sigma_{k}$ and the value of $\sigma_{f}^{2}$ of no spatial correlation $(a=0.0)$ is considerably larger than that of considered spatial correlation $(a \neq 0.0)$. In all cases of $a$, $\sigma_{f}^{2}$ is the very small. The reason for this is that $Q_{m}$ may dominantly depend on the pattern of scattering lower permeability cells and/or arrangement of lower permeability cells.

\section{Conclusions}

With generation of heterogeneous random permeability fields, solving the 2-D steady state seepage flow problem by the finite element method has been attempted. Parametric studies have been performed to show the effect of the standard deviation and the correlation distance of soil permeability on the total flow rate in a confined model. The results are compared with the theoretical solution. The conclusions drawn from this study are as follows:

1. The simulation of random permeability fields can reproduce realizations using its basis statistics (mean, variance and spatial correlation).

2. The mean flow rate $Q_{m}$ reduces rapidly from the theoretical flow rate $Q_{t}$ as the global standard deviation $\sigma_{k}$ increases. And, as correlation distance $a$ increases, $Q_{m}$ becomes far from $Q_{t}$. The explanation lies in the fact that flow rate is greatly influenced by the existence of lower permeability areas.
3. For various values of $a$, the variance of flow rate $\sigma_{f}^{2}$ is the very small even with large global standard deviation $\sigma_{k}$.

\section{Acknowledgement}

This research is funded by Grant-in-Aid for Scientific Research (B)(1) (Project Number 12556037) of the Ministry of Education, Science, Sports and Culture, Japan. The authors wish to thank for its generous financial assistance.

\section{References}

[1] Fenton, G. A. and Griffiths, D. V. (1996): Statistics of free surface flow through stochastic earth dam, J. Geotechnical Engineering, ASCE, 122(6), pp.427436.

[2] Fenton, G. A. and Vanmarcke, E. H. (1990): Simulation of random fields via local average subdivision, J. Engineering Mechanics, ASCE, 116(8), pp.17331749.

[3] Freeze, R. A. (1975): A stochastic-conceptual analysis of one-dimensional groundwater flow in nonuniform homogeneous media, Water Resources Research, 11(1), pp.725-741.

[4] Griffiths, D. V. and Fenton, G. A. (1993): Seepage beneath water retaining structures founded on spatially random soil, Geótechnique, 43(4), pp.577-587.

[5] Griffiths, D. V. and Fenton, G. A. (1997): Threedimensional seepage through spatially random soil, $J$. Geotechnical and Geoenvironmental Engineering, 123(2), 118(10), pp.153-160.

[6] Hamaguchi, T. (1998): Studies on Inverse Problems Relating to Design for Underground Dam through New Modeling for Groundwater Flow with Moving Boundaries, Doctoral dissertation, Kyoto University, pp.91-98.

[7] Hoeksema, R. J. and Kitanidis, P. K. (1985): Analysis of the spatial structure of properties of selected aquifers, Water Resources Research, 21(4), pp.563572.

[8] Journel, A. G. and Huijbregts, C. J. (1978): Mining Geostatistics, Academic, San Diego, Calif., pp.161195.

[9] Smith, L. and Freeze, R. A. (1979): Stochastic analysis of steady state groundwater flow in a bounded domain - 1. One-dimensional simulations, Water Resources Research, 15(3), pp.521-528.

[10] Sudicky, E. A. (1986): A natural gradient experiment on solute transport in a sand aquifer: Spatial variability of hydraulic conductivity and its role in the dispersion process, Water Resources Research, 22(13), pp.2069-2082.

[Discussion open until June 30, 2002] 